\title{
APLICAÇÃO DE ETEFOM SOBRE O PLENO FLORESCIMENTO EM PESSEGUEIRO DA CV. OKINAWA
}

\author{
${ }^{1}$ Iohann Metzger Bauchrowitz, 2 Tracy Paola Maçaneiro \\ ${ }^{1}$ Msc. Engenheiro Agronomo; ${ }^{2}$ Graduanda em Agronomia -Centro de Ensino Superior dos Campos Gerais - CESCAGE.
}

RESUMO: O pessegueiro é a principal frutífera de caraço produzida no Brasil, sendo uma cultura comum na região sul do Brasil, entretando seu cultivo, apresenta limitação pela falta de mão de obra para realização de práticas culturais, sendo a principal o raleio dos frutos. Este trabalho teve como objetivo testar diferentes concentrações de Etefom sobre o florescimento de pessegueiro. O experimento foi realizado em Delineamento de blocos casualizados (DBC) composto de 6 tratamentos e 5 repetições cada, cada uma das repetições foi formada por 5 plantas. Os tratamento realizados nos frutos foram T1 (Testemunha sem aplicação de Etefom) T2 ( 25 ppm de Etefom), T3 (50 ppm de Etefom), T4 (100 ppm de Etefom), T5 (150 ppm de Etefom) e T6 (200 ppm de Etefom), as análises realizadas nos frutos foram queda de flores $(\%)$, diâmetro $(\mathrm{mm})$ e massa dos frutos $(\mathrm{g})$, sólidos solúveis ( ${ }^{\circ}$ Brix), acidez titulável $(\%)$ e ratio. As concentrações de Etefom apresentaram resultados positivos na qualidade física dos frutos, enquanto que não houve efeito sobre as qualidades químicas. $\mathrm{O}$ uso de etefom é recomendado para realização do raleio nos frutos de pessegueiro, sendo a queda das flores e aumento do diâmetro dos frutos os principais resultados positivos.

Palavras-chave: Raleio, qualidade, frutos.

\section{APPLICATION OF ETEFOM ON THE FULL BLOOMING IN PESSEGUEIRO DA CV. OKINAWA}

ABSTRACT: The peach tree is the main fructiferous fruit produced in Brazil, being a common crop in the southern region of Brazil, amidst its cultivation, is limited by the lack of manpower to carry out cultural practices, the main being the fruit thinning. The objective of this work was to test different concentrations of Etefom on the flowering of peach trees. The experiment was carried out in a randomized block design (DBC) composed of 6 treatments and 5 replicates each, in each of the replicates was formed by 5 plants. The treatments performed on fruits were T1 (Witness without application of Etefom) T2 (25 ppm Etefom), T3 (50 ppm Etefom), T4 (100 ppm Etefom), T5 (150 
ppm Etefom) and T6 (\%), diameter ( $\mathrm{mm}$ ) and fruit mass (g), soluble solids ( ${ }^{\circ}$ Brix), titratable acidity (\%) and ratio were evaluated. The Etefom concentrations showed positive results in the physical quality of the fruits, while there was no effect on the chemical quality. The use of etefom is recommended for thinning of peach fruit, with flowers falling and fruit diameter being the main results.

Keywords: Scaly, quality, fruits.

\section{INTRODUÇÃO}

O aumento na procura de frutas para uma alimentação saudável deve como efeito a elevação na importação de frutíferas, as quais o Brasil não apresenta autossufiência de produção necessitando de importação dos países vizinhos principalmente Argentia e Chile nas frutíferas de clima temperado (SEAB/ DERAL, 2016).

As frutíferas conhecidas como frutas de caroços, pêssego, ameixa e nectarina apresentam-se ricas em fontes de vitaminas e minerais sendo seu consumo indicado para a população (CARDOSO et al., 2010), entretanto estas frutíferas apresentam limitações de cultivo no Brasil, sendo o clima o fator limitante para a expansão da cultura em diferentes regiões (HAWERROTH et al., 2010).

Nas regiões produtoras como a falta e os custo de mão de obra para as atividades nos pomares são os principais fatores que encarencem os custos de produto elevando o valor final dos produtos, e dificultando a expansão das áreas cultivadas nas regiões produtoras (AHRENS et al., 2014), sendo principalmente os estados do Sul do Brasil os maiores produtores destas frutíferas.

Entre as principais atividades que demanda uma elevada quantidade de mão de obra, e que são indispensáveis para a produção de frutos de pessegueiros com qualidade e o raleio dos frutos, o qual existem várias formas de realização, sendo a mais utilizadas nos dias atuais o raleio químico de frutos (PAVANELLO; AYUB, 2012). Entratando o raleio manual pode ser realizado de forma mais eficiente, porém de uma forma mais lenta e com maior uso de mão de obra (CARVALHO et al., 2015; BAUCHROWITZ et al., 2016). 
A qualidade de frutos de pessegueiro (Prunus persica L), assim como ocorre em outras frutíferas apresentam respostas em relação aos tratos culturais, visando melhoria na qualidade dos frutos, entre estes tratos podemos mencionar a utilização do raleio dos frutos (CAVALLARI, 2013) e esta resposta afeta diretamente a qualidade comercial dos frutos e a rentabilidade do produtor (EMATER-PR, 2001).

Os efeitos dos diferentes produtos químicos sobre o raleio dos frutos podem variar de que acordo com o produto utilizado para a realização do raleio dos frutos, ou em relação à forma e momento de aplicação do produto, sendo possível que em concentrações elevadas ocorra a perda de grande parte da produção causando (PAVANELLO; AQYUB 2014).

Este trabalho teve como objetivo, testar diferentes concentrações de Etefom com raleante no pleno florescimento em frutos de pessegueiro da cultivar okinawa, e os efeitos da aplicação sobre o diametro dos frutos, massa dos frutos, Solidos soluveis totais, acidez titulavel e ratio.

\section{MATERIAL E MÉTODOS}

O experimento foi realizado no municipio de Palmeira-Pr, durante a safra 2016/2017, o clima da região sendo koppen e classificado com Cfb subtropical umido com temperatura média de $18^{\circ} \mathrm{C}$ e preciptação média anual proxima de $1500 \mathrm{~mm}$ (IAPAR, 2000).

A cultivar de pessegueiro utilizada para este experimento foi a Okinawa plantadas no ano de 2010, conduzidos em espaçamento de 1,5 metros entre plantas e 5 metros entre linhas em formato de $Y$.

O experimento foi realizado em Delineamento de blocos casualizados (DBC) composto de 6 tratamentos e 5 repetições cada, em cada uma das repetições foi formada por 5 plantas. Os tratamentos realizados nos frutos foram T1 (Testemunha sem aplicação de Etefom) T2 (25 ppm de Etefom), T3 (50 ppm de Etefom), T4 (100 ppm de Etefom), T5 (150 ppm de Etefom) e T6 (200 ppm de Etefom), o volume da calcada aplicada sobre as plantas foi de 2 litros de calca + produto por parcela, utilizando pulverizador costal. 
Para as avaliações da \% de queda das flores foram selecionadas 200 flores que foram contadas no pleno florescimento e 30 dias antes da colheita dos frutos, frutos foram analisados o diâmetro $(\mathrm{mm})$ com o auxilio de paquímetro manual, massa dos frutos (g) com o auxilio de balança de precisão, sólidos solúveis ( $\left.{ }^{\circ} B r i x\right)$ com o auxilio de refratómetro modelo (RT-90), acidez titulável (\%) através da equação disponível na metodologia de Bauchrowitz et al. (2018) e ratio através da relação SS/AT. Os dados foram submetidos à análise de regressão a $5 \%$, pelo programa SISVAR 5.6 (FERREIRA, 2014).

\section{RESULTADOS}

As diferentes concentrações de Etefom aplicadas sobre o pleno florescimento em pessegos da cultivar Okinawa (Prunus persica L.) afetam diretamenta o número de flores caidas durante o ciclo da planta, causado pela absição de flores. As concentrações superiores a $100 \mathrm{ppm}$ aplicados no pleno florescimento causaram redução no florescimento superior a $58,20 \%$ se comparado com os valores iniciais antes da aplicação do produto, concentrações de 50 ppm causaram uma absição de $51,60 \%$ das flores (Gráfico 1).

Gráfico 1. Queda de flores, em relaçao a diferentes concentraçoes de Etefom aplicados em pleno florescimento.

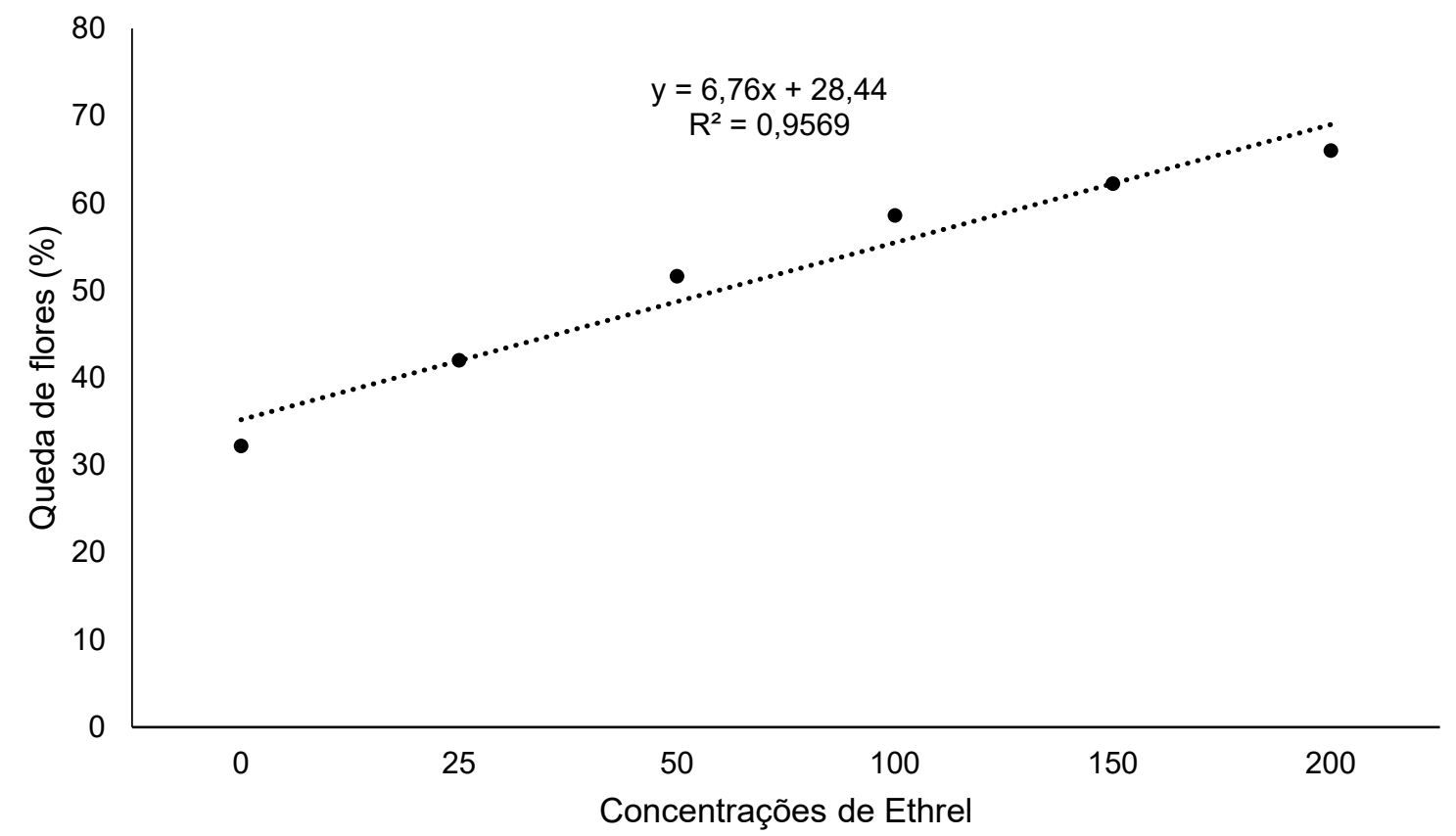


Quando se utiliza o produto Etefom para a realização do raleio de flores, os valores com maiores índices de queda são os que apresentam melhores resultados necessitando uma menor necessidade de raleio manual dos frutos posteriormente.

Com a redução causada no número de flores permanentes nas plantas, após a aplicação do Etefom, houve um efeito benéfico na qualidade de frutos com um aumento no diâmetro dos frutos e massa dos frutos, este aumento positivo ocorreu pela redução na competição entre os frutos permanecentes por espaço, luz e fotoassimilados para seu desenvolvimento.

O diâmetro dos frutos apresentaram aumento quando comparados aos valores obtidos no tratamento T1 e outras concentrações, este aumento no diâmetro causam melhorias nos parâmetros de classificação dos frutos, tornando-se uma prática como a realização do raleio de frutos, principalmente pelos benefícios comerciais e por se efetar a desuniformidade de produção entre diferentes anos de cultivo (Gráfico 2).

As concentrações de Etefom de 100 e 150 ppm aplicados não apresentaram diferenças entre si nos valores de diâmetro dos frutos, sendo obtidos nas duas concentrações frutos com diâmetro de 49,00 mm aproximadamente, enquanto no tratamento com concentração de 200 ppm os valores obtidos nos frutos foram de $51,80 \mathrm{~mm}$. 


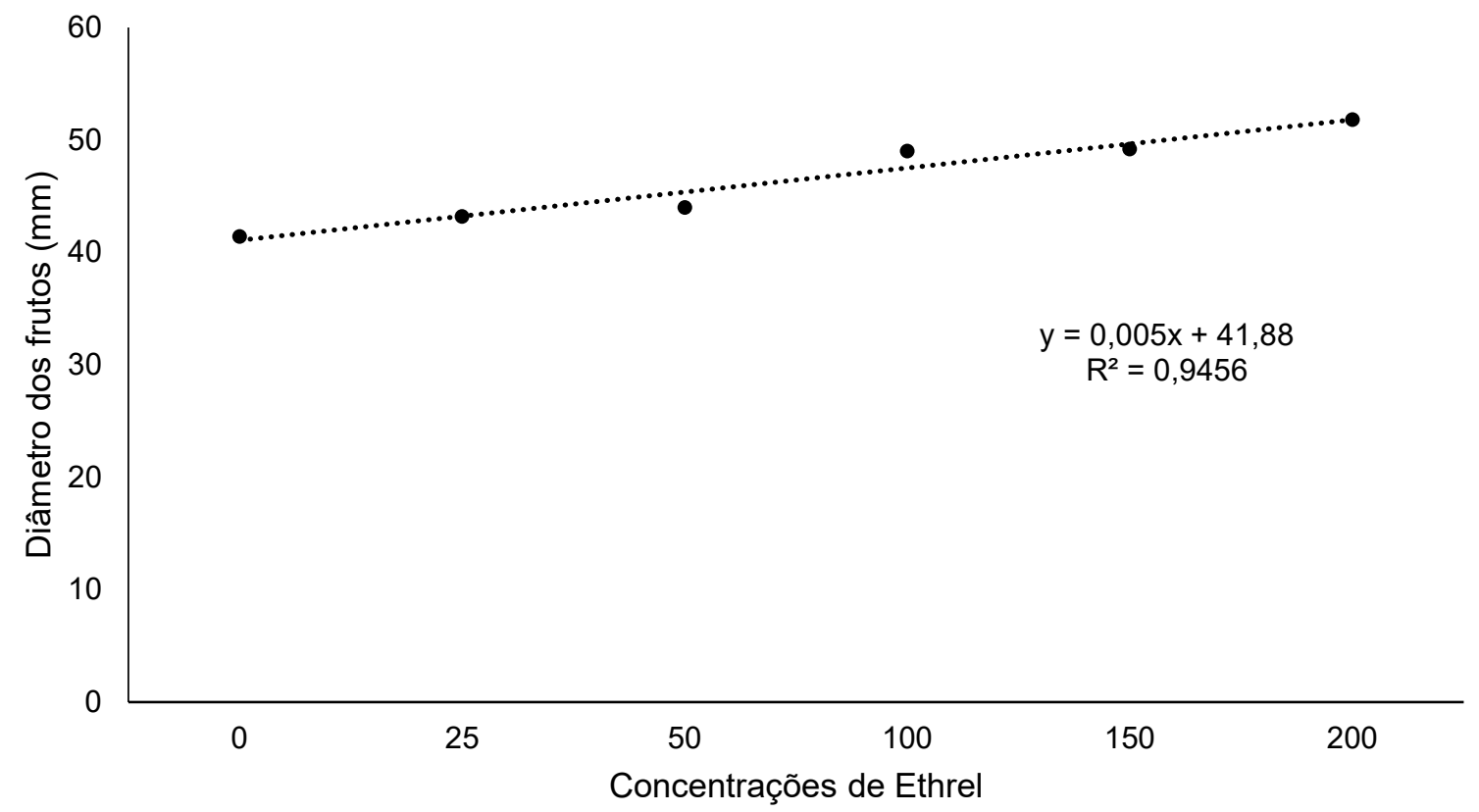

Gráfico 2. Diâmetro dos frutos, em relaçao a diferentes concentraçoes de Etefom aplicados em pleno florescimento

Assim como ocorrido no diâmetro dos frutos, a massa dos frutos apresentaram resultados positivos com a aplicação do Etefom sobre o pleno florescimento, com objetivo de realização do raleio dos frutos.

Como ocorrido no diâmetro dos frutos, as concentraçoes de 100 e 150 ppm aplicadas não apresentaram diferenças entre as massa dos frutos, sendo obtidos valores de 81,00 g neste dois tratamentos, já quando a concentração de 200 ppm de Etefom aplicado no pleno florescimento os valores de massa dos frutos obtidos no momento da colheita foram de $88,00 \mathrm{~g}$ (Gráfico 3). 


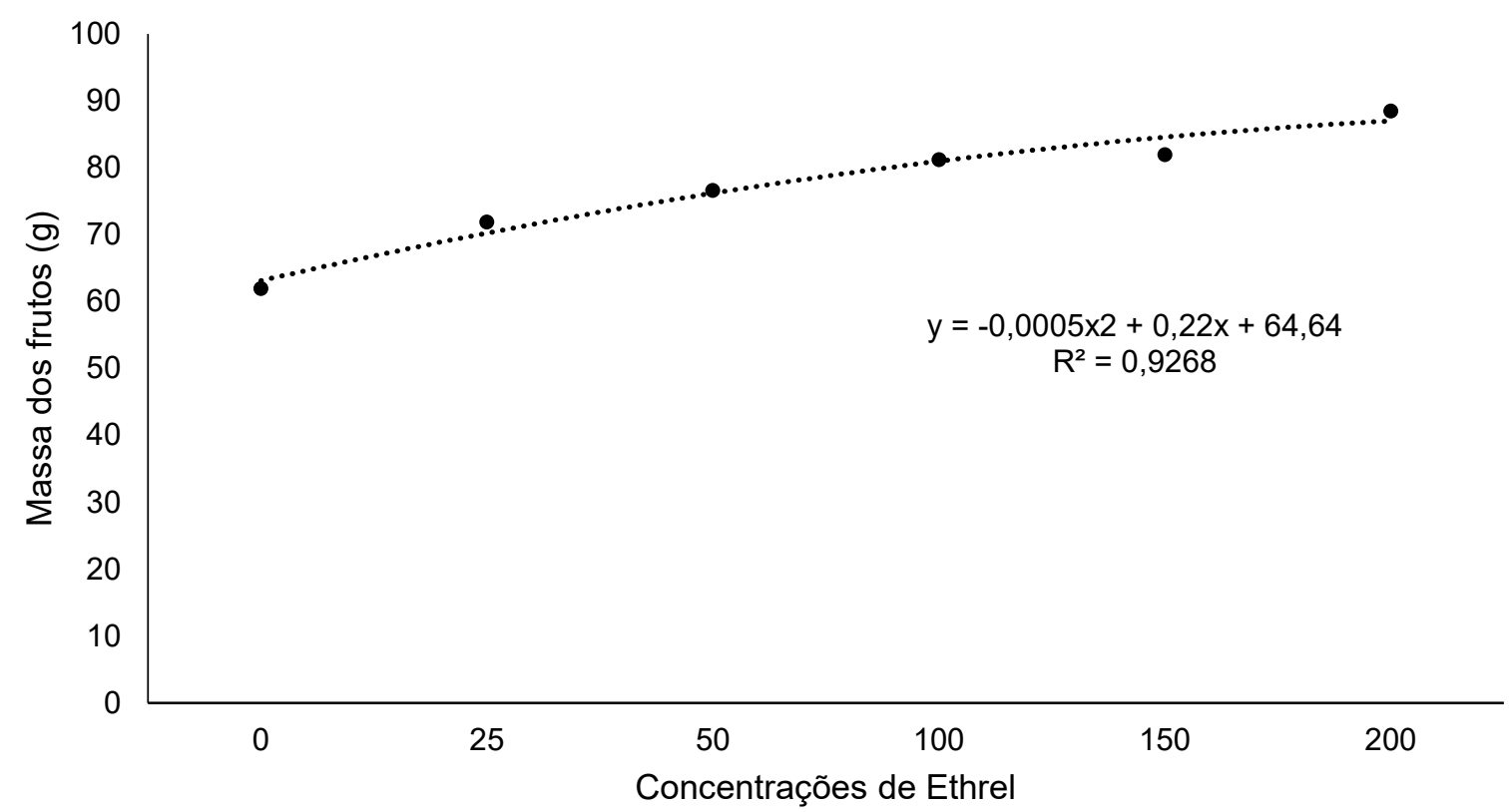

Gráfico 3. Massa dos frutos, em relaçao a diferentes concentraçoes de Etefom aplicados em pleno florescimento.

O efeito da aplicação do Etefom sobre o pleno florescimento não afetaram as qualidades quimícas dos frutos de sólidos solúveis (SS), os quais em todos os tratamentos apresentaram valores de aproximadamente $12,50^{\circ} \mathrm{Brix}$, enquanto a quantidade de acidez titulável (AT) nos frutos de todos os tratamentos foram de $0,35 \%$, o ratio dos frutos de modo geral foi de 35,71 sendo que estes valores são influenciados diretamente pelo SS e AT.

A aplicação do Etefom sobre as qualidades químicas dos frutos não apresentou resultados, ocorrendo devido estas qualidades não serem definidas no momento do florescimento e sim durante o ciclo da cultura e seus valores são definidos geneticamente pelas cultivares.

\section{DISCUSSÃO}

O raleio de frutos e flores é um trato cultural quase que obrigatório realizado pelos produtores, visando a melhor classificação comercial dos frutos (PAVANALLE; 
AYUB, 2012), o principal efeito do raleio dos frutos é o aumento do diâmetro dos frutos como é possivel observar em frutos de ameixeira japonesa (BAUCHROWITZ et al., 2018).

Fruto dos genótipos G32 e G35 apresentam diâmetros superiores a 46,90 mm (BAUCHROWITZ et al., 2018), em frutos de a cultivar Aurora apresentaram diâmetro de 54,00 mm (MARTINS et al., 2013), e na cultivar Maciel de aproximadamente 55,00 mm (SEIBERT et al., 2010).

Outros efeitos do raleio podem ser observados em frutos de macieiras submetidas ao raleio de flores utilizando-se spray de água em diferentes pressões, causando uma retirada das flores da plantas (AWAD, 2006), além do efeito no diâmetro dos frutos, outras características afetadas pelo raleio dos frutos é a massa dos frutos, assim como ocorreu no experimento com pessegueiros.

Este efeito na massa dos frutos ocorre devido a retirada do excesso de frutos produzidos no pleno florescimento, favorecendo um desenvolvimento uniforme e maior expansão e celular, o qual afeta o diâmetro dos frutos e a massa dos frutos pelo maior fluxo de fotoassimilados até os frutos permanecentes (KERBAUY, 2004), este efeito observado em frutos da cultivar Diamante, a massa apresentou influência relacionada aos tratamentos e a maturação dos frutos (PÉREZ-LÓPES et al., 2014).

Em frutos de macieira é possivel observar o aumento na massa de frutos quando comparados às diferentes concentrações de 6-benziladenia, em concentrações de 80 e $120 \mathrm{mg} / \mathrm{l}^{-1}$ apresentaram os melhores resultados de massa, quando aplicados sobre frutos em desenvolvimento de $5^{\mathrm{a}} 10 \mathrm{~mm}$ (PETRI et al., 2013), quando aplicado as diferentes concentrações de Promallin, os melhores resultados obtidos em relação à massa dos frutos foram na concentração de $0,5 \mathrm{~L}$ ha $^{-1}+$ Maxcel 2,0L ha-1 aplicados em frutos de 10-15 mm (PETRI et al., 2016).

Na cultivar Oro B a massa dos frutos obtida no momento da colheita foi variando entre 63,0 a $105,01 \mathrm{~g}$, ja na cultivar Flordaking a massa variou entre 87,00 a 124,70 g (QUEIROZ 2014), na cultivar chimarrita a massa variou no momento da colheita entre 104,40 a 119,20 g (PICOLOTTO et al., 2009). 
Em pessegueiros é possivel observar as variações entre as cultivares nos valores de Sólidos Solúveis, entretanto possuem redução quando armazenados no momento da colheita, não apresentando-se influenciados por tratamento químicos (JIN et al., 2014), assim como observado em frutos da cultivar Sensação em que não se observa variações na qualidade química dos frutos influenciados pelos tratamentos aplicados sobre os frutos (SILVA et al., 2016), os frutos das cultivares Early Rich e Elegant Lady apresentaram valores de Solidos Sóluveis proximos de 11,00 ${ }^{\circ}$ Brix (CANO-SALAZAR et al., 2012),

Os valores de AT em frutos de pessegueiro assim como ocorrido nos Sólidos Sóluveis não apresentaram diferenças influenciadas pelos tratamentos químicos aplicados sobre o pleno florescimento (OLIVEIRA,CEREDA, 2003), este efeito é observado em fruto das cultivares Royal Glory e Sweet Dream, a qual apresentam valores de AT de 3,80\% para as duas cultivares (CANO-SALAZAR et al., 2012), enquanto na cultivar Flavorcrest foi de 1,03\% (MINAS et al., 2015).

\section{CONCLUSÕES}

A aplicação de Etefom no pleno florescimento apresenta resultados positivos, sendo recomendada a utilização deste produto como raleante em frutos de pessegueiros.

As concentrações superiores a 100 ppm apresentam resultados positivos e significativos na queda de flores, e melhoria nas caracteristicas de diâmetro e massa dos frutos.

\section{REFERÊNCIAS}

AHRENS, R. B.; PAVANELLO, A. P.; AHREN, D. C.; FRANCISCO, A. C.; AYUB, R. A. ANÁLISE ECONÔMICA DO RALEIO QUÍMICO E MANUAL EM AMEIXEIRAS. Asociación Interciencia, Caracas, Venezuela, v. 39, n. 10, p.723-726, out. 2014. 
AWAD. M. A. Water spray as a potential thinning agent for date palm flowers (Phoenix dactylifera L.) c.v. 'Lulu'. Scientia Horticulturae, [s.I.], v. 111, n. 1, p.44-48, dez. 2006. Elsevier BV. http://dx.doi.org/10.1016/j.scienta.2006.09.005.

BAUCHROWITZ, I. M.; SILVA, C. M.; FRANCISCO, A. L. O.; MAÇANEIRO, T. P. EFEITO DO RALEIO MANUAL EM FRUTOS DE AMEIXA JAPONESA (PRUNUS SALICINA LIND) SOBRE DIFERENTES POPULAÇÕES. Revista Científica Rural, Bagé-rs, v. 20, n. 1, p.72-79, maio 2018.

BAUCHROWITZ, I. M.; SILVA, C. M.; KITZBERGER, C. S. G.; EILERT, J. B.; NETO, J. S.; FRANCISCO, A. L. O. Avaliação sobre o efeito de diferentes épocas de raleio manual em ameixa japonesa (Prunus salicina lind). Scientia Rural, Ponta Grossa-pr, v. 14, n. 1, p.52-64, dez. 2016.

BAUCHROWITZ, I. M.; SILVA, C. M.; KITZBERGER, C. S. G.; EILERT, J. B.; NETO, J. S.; FRANCISCO, A. L. O. AVALIAÇÃO SOBRE O EFEITO DE DIFERENTES ÉPOCAS DE RALEIO MANUAL EM AMEIXA JAPONESA

BAUCHROWITZ, I. M.; SILVA, C. M.; MAÇANEIRO, T. P. AVALIAÇÃO DE CARACTERÍSTICAS FENOLÓGICAS E PRODUTIVAS DE QUINZE GENÓTIPOS DE PESSEGUEIRO DO BANCO DE GERMOPLASMA DO IAPAR. Revista Científica Rural, Bagé-rs, v. 20, n. 1, p.1-14, maio 2018.

CANO-SALAZAR, J.; ECHEVERRÍA, G.; CRISOSTO, C. H.; LOPEZ, L. Cold-Storage Potential of Four Yellow-Fleshed Peach Cultivars Defined by Their Volatile Compounds Emissions, Standard Quality Parameters, and Consumer Acceptance. Journal Of Agricultural And Food Chemistry, [s.I.], v. 60, n. 5, p.1266-1282, 8 fev. 2012. American Chemical Society (ACS). http://dx.doi.org/10.1021/if204126m.

CARDOSO, W. S.; PINHEIRO, F. A.; PEREZ, R.; PATELLI, T.;FARIA,E. R. Desenvolvimento de uma salada de frutas: da pesquisa de mercado à tecnologia de alimentos: Fruit salad development: from market research to food technology. Ciência e Tecnologia de Alimentos, Campinas, v. 30, n. 2, p.151-162, jun. 2010.

CARVALHO, R. I. N.; SORRENTI, G.; PEREIRA, G. P.; VENDRAMIN, D. W.; OLIVEIRA, R. F.; KUSSLER, R. E. PODA VERDE E QUALIDADE DO FRUTO DA AMEIXEIRA EM ALTA DENSIDADE. R. Eletr. Cient. Uergs, Porto Alegre-rs, v. 1, n. 1, p.69-73, dez. 2015.

CAVALLARI, Ludmilla de Lima. RALEIO DE FRUTOS EM VARIEDADES DE LICHIEIRA. 2013. 60 f. Tese (Doutorado) - Curso de Agronomia, (produção Vegetal)., Faculdade de Ciências Agrárias e Veterinárias - Unesp, Câmpus de Jaboticabal,, Jaboticabal, 2013. 
EMATER-PR. Colheita, classificação, Embalagem, acondicionamento e armazenamento da ameixeira e nectarina por Fukuo Morimoto, Curitiba, 2001. 20 p. (Informação Técnica, 61).

FERREIRA, D. F. Sisvar: a Guide for its Bootstrap procedures in multiple comparisons. Ciênc. agrotec. [online]. 2014, vol.38, n.2 [citado 2015-10-17], pp. 109-112. Disponible en: ISSN 1413-7054. http://dx.doi.org/10.1590/S1413-70542014000200001.

HAWERROTH, F. J.; HERTER, F. G; PETRI, J.L; LEITE, G. B; PEREIRA, J.F.M. Dormência em frutíferas de clima temperado. Pelotas-RS : Embrapa Clima Temperado, 2010. 56 p.

IAPAR - Instituto Agronômico do Paraná. Cartas Climáticas do Paraná. Versão 1.0.2000.

JIN, P.; ZHU, H.; WANG, L.; SHAN, T.; ZHENG, Y. Oxalic acid alleviates chilling injury in peach fruit by regulating energy metabolism and fatty acid contents. Food Chemistry, [s.I.], v. 161, p.87-93, out. 2014. Elsevier BV. http://dx.doi.org/10.1016/j.foodchem.2014.03.103..

KERBAUY, G. B. Fisiologia Vegetal. Rio de Janeiro. Ed. Guanabara Koogan, 439p. 2004.

MARTINS, R. N.; MATTIUZ, B.; SANTOS, L. O.; et al. Estádios de maturação de pêssegos 'Aurora-1' para o processamento mínimo. Revista Brasileira de Fruticultura, [s.I.], v. 35, n. 2, p.391-397, jun. 2013. FapUNIFESP (SciELO). http://dx.doi.org/10.1590/s0100-29452013000200008.

MINAS, I. S.; FORCADA, C. F.; DANGL, G. S.; GRADZIEL, T. M.; DANDEKAR, A. M.; CRISOSTO, C. H. Discovery of non-climacteric and suppressed climacteric bud sport mutations originating from a climacteric Japanese plum cultivar (Prunus salicina Lindl.). Frontiers In Plant Science, [s.I.], v. 6, p.1-10, 12 maio 2015. Frontiers Media SA. http://dx.doi.org/10.3389/fpls.2015.00316.

OLIVEIRA, M. A.; CEREDA, M. P. Pós-colheita de pêssego (Prunus pérsica L.Bastsch) revestidos com filmes a base de amido como alternativa á cera comercial. Ciência Tecnologia Alimentar, Campinas, v.23, n. seplemntar, p. 28-33, 2003

PAVANELLO, A. P.; AYUB, R. A. Aplicação de ETEFOM no raleio químico de ameixeira e seu Efeito Sobre a Produtividade. Revista Brasileira de Fruticultura, Jaboticabal-SP, v.34, n.1, p.309-316, 2012.

PAVANELLO, A. P.; AYUB, R. A. Raleio químico de frutos de ameixeira com ETEFOM. Ciência Rural, [s.I.], v. 44, n. 10, p.1766-1769, out. 2014. FapUNIFESP (SciELO). http://dx.doi.org/10.1590/0103-8478cr20131097. 
PÉREZ-LÓPEZ, A.; CHÁVEZ-FRANCO, S. H.; VILLASENÕR-PEREA, C. A.; ESPINOSA-SOLARES, T.; HERNÁNDEZ-GÓMEZ, J. H.; LOBATO-CALLEROS, C. Respiration rate and mechanical properties of peach fruit during storage at three maturity stages. Journal Of Food Engineering, [s.I.], v. 142, p.111-117, dez. 2014. Elsevier BV. http://dx.doi.org/10.1016/j.jfoodeng.2014.06.007.

PETRI, J. L.; LEITE, G. B.; FRANCESCATTO, P.;GABARDO, G. C.; SEZERINO, A. A. Raleio químico de floração com complemento de pós floração na macieira grupo 'gala'. Revista Eletrônica Cientifica Uergs, Porto Alegre, v. 2, n. 2, p.175-181, ago. 2016.

PETRI, J. L.; HAWERROTH, F. J.; LEITE, G. B.;COUTO, M. RALEIO QUÍMICO EM MACIEIRAS 'FUJI SUPREMA' E 'LISGALA'. Revista Brasileira de Fruticultura, Jaboticabal - Sp, v. 35, n. 1, p.170-182, mar. 2013.

PICOLOTTO, L.; MANICA-BERTO, R.; PAZIN, D.; et al. Características vegetativas, fenológicas e produtivas do pessegueiro cultivar Chimarrita enxertado em diferentes porta-enxertos. Pesq. Agropec. Bras., Brasília, v. 44, n. 6, p.583-589, jun. 2009.

QUEIROZ, H. T. Caracterização de genótipos de pessegueiros e ameixeiras na depressão central do estado do Rio Grande do Sul. 2014. 82 f. Dissertação (Mestrado) - Curso de Agronomia, Fitotecnia, Universidade Federal do Rio Grande do Sul Faculdade de Agronomia, Porto Alegre -Rs, 2014.

SEAB/DERAL. SEAB - Secretaria de Estado da Agricultura e do Abastecimento DERAL- Departamento de Economia Rural. Fruticultura 2015. Disponível em : http://www.agricultura.pr.gov.br/arquivos/File/deral/Prognosticos/fruticultura 201415 .pdf . Acesso: 30 abril de 2018.

SEIBERT, E.; LEÃO, M. L.; RIETH, S.; et al . Efeitos do condicionamento na qualidade de pêssegos Maciel. Acta Scientiarum. Agronomy, [s.I.], v. 32, n. 3, p.477-483, 27 ago. 2010. Universidade Estadual de Maringa.

http://dx.doi.org/10.4025/actasciagron.v32i3.3947.

SILVA, P. S.; BARRETO, C. F.; MORENO, M. B.; SCHIAVON, A. V.; FARIAS, P. C. M.; FACHINELLO, J. C. PÊSSEGOS 'SENSAÇÃO' SUBMETIDOS INOCULAÇÃO DE MONILINIA FRUCTICOLA E APLICAÇÃO DE ÁCIDO SALICÍLICO EM PÓSCOLHEITA. Rev. Iber. Tecnología Postcosecha, Hermosillo, México, v. 17, n. 2, p.241-246, dez. 2016 